PROCEEDINGS OF THE

AMERICAN MATHEMATICAL SOCIETY

Volume 135, Number 4, April 2007, Pages 961-968

S 0002-9939(06)08546-7

Article electronically published on September 26, 2006

\title{
GENERALIZATIONS OF CAYLEY'S $\Omega$-PROCESS
}

\author{
WALTER FERRER SANTOS AND ALVARO RITTATORE
}

(Communicated by Martin Lorenz)

\begin{abstract}
In this paper we axiomatize some constructions and results due to Cayley and Hilbert. We define the concept of $\Omega$-process for an arbitrary affine algebraic monoid with zero and unit group $G$. In our situation we show how to produce from the process and for a linear rational representation of $G$ a number of elements of the ring of $G$-invariants $S(V)^{G}$ that is large enough to guarantee its finite generation. Moreover, using complete reducibility, we give an explicit construction of all $\Omega$-processes for reductive monoids.
\end{abstract}

\section{Preliminaries}

We assume throughout that the base field $\mathbb{k}$ is algebraically closed of characteristic zero and that all the geometric and algebraic objects are defined over $\mathbb{k}$. A linear algebraic monoid is an affine algebraic variety $M$ with an associative product $M \times M \rightarrow M$ which is a morphism of algebraic $\mathbb{k}$-varieties, and that is also equipped with a neutral element $1 \in M$ for this product. In this situation, it can be proved that the group of invertible elements of $M$ (called the unit group of $M$ and denoted by $G(M)=\left\{g \in M: \exists g^{-1}\right\}$ ) is an affine algebraic group that is open in $M$. In [3] and [4] the reader will find two extensive surveys on the topic of linear algebraic monoids.

Also in this paper we will add the following density condition: $G(M)$ is a dense subset of $M$. This will allow us to have a better control of the representations of $M$ in terms of those of $G(M)$.

A linear algebraic monoid $M$ is a reductive monoid if its unit group is reductive (and dense in $M$, in accordance with the density condition).

An arbitrary algebraic monoid $M$ with unit group $G$ supports an action of $G \times G$, given by $(a, b) \cdot m=a m b^{-1}$; in $[5$, Thm. 1] it is proved that this action has $G$ as an open orbit.

The following notation will be in force in this article.

If $M$ is an irreducible reductive monoid with unit group $G=G(M)$, we call $T$ a maximal torus of $G, B$ a Borel subgroup containing $T, B^{-}$its opposite Borel subgroup, $\mathcal{X}(T)$ the set of weights and $\mathcal{X}_{+}(T)$ the semigroup of dominant weights with respect to $B$. If $\lambda \in \mathcal{X}_{+}(T)$ is a dominant weight, then $V_{\lambda}$ will denote the irreducible $G$-module associated to $\lambda$.

Received by the editors August 30, 2005 and, in revised form, October 31, 2005.

2000 Mathematics Subject Classification. Primary 20G20, 16W22, 14Lxx.

The first author would like to thank Csic-UDELAR and Conicyt-MEC.

The second author would like to thank FCE-MEC, project number 10018.

(C)2006 American Mathematical Society Reverts to public domain 28 years from publication 
If $V$ is a rational representation of $G$, we denote by $S(V)$ the symmetric algebra built on $V$.

\section{REPRESENTATIONS AND CHARACTERS OF ALGEBRAIC MONOIDS}

If $V$ is a finite-dimensional $\mathbb{k}$-space, we say that an action $M \times V \rightarrow V$ is polynomial if the associated map $M \rightarrow \operatorname{End}_{\mathbb{k}}(V)$ is a morphism of affine algebraic monoids; in particular in the case that $M$ is endowed with $0 \in M, 0 \cdot v=0$ for all $v \in V$.

Observe that the action map $M \times V \rightarrow V$ induces a morphism of $\mathbb{k}$-spaces $\chi_{V}: V \rightarrow V \otimes \mathbb{k}[M]$ that is a counital comodule structure on $V$. The relationship between the structure $\chi_{V}$ and the action of $M$ is given as follows: $\chi_{V}(v)=\sum v_{0} \otimes v_{1}$ if and only if for all $m \in M, m \cdot v=\sum v_{1}(m) v_{0}$ (Sweedler's notation). In this paper we consider in general the actions on the left side, but it is clear that all the concepts we deal with have a formulation for a right action.

If $V$ is a polynomial $M$-module, we will denote as $V^{M}$ the subspace of $M$ invariants; it follows easily that $V^{M}=\left\{v \in V: \chi_{V}(v)=v \otimes 1\right\}$.

It is easy to prove, in the same way as for affine algebraic groups and their representations (see for example [1]), that the above correspondence is an isomorphism between the category of finite-dimensional polynomial $M$-modules and the category of finite-dimensional counital comodules for the bialgebra $\mathbb{k}[M]$.

If we drop the hypothesis on the dimension, we obtain the concept of a general polynomial $M$-module that is simply an arbitrary counital comodule $V$ for the bialgebra $\mathbb{k}[M]$; the comodule structure in $V$ will be denoted as $\chi_{V}: V \rightarrow V \otimes \mathbb{k}[M]$. This corresponds to a vector space $V$ endowed with a locally finite polynomial action of $M$. In other words the action of $M$ on $V$ is such that any element $v \in V$ is contained in a finite-dimensional $M$-stable polynomial submodule $V_{v} \subset V$.

Definition 2.1. Let $M$ be a linear algebraic monoid. A polynomial character of $M$ is a multiplicative morphism $\rho: M \rightarrow \mathbb{k}$ such that $\rho(1)=1$. We will denote as $\mathcal{X}(M)$ the abstract monoid of all characters of $M$.

We say that the character $\rho$ is trivial if it only takes the value $1 \in \mathbb{k}$.

Observation 2.2. (1) The description of $\mathrm{GL}_{n}$ as the set of matrices with nonzero determinant can be generalized to general affine monoids. Indeed, if $M$ is an arbitrary affine monoid, as is well known (see for example [3]) we can suppose that $M$ is a closed submonoid of $\mathrm{M}_{n}(\mathbb{k})$ for some $n$ and with $G(M)=M \cap \mathrm{GL}_{n}(\mathbb{k})$. Hence the restriction of det to $M$ is a character, and

$$
G(M)=M_{\text {det }}=\{m \in M: \operatorname{det}(m) \neq 0\} .
$$

(2) Observe that if $0 \in M$ and $\rho$ is a nontrivial polynomial character, then $\rho(0)=\rho(0 m)=\rho(0) \rho(m)$ for all $m \in M$, and thus $\rho(0)=0$.

Observation 2.3. Assume that $V$ is a polynomial $M$-module and call $G=G(M)$ the group of invertible elements. It is clear that the restriction of the action from $M$ to $G$ makes of $V$ a rational $G$-module. If $\chi$ is the associated $\mathbb{k}[M]$-comodule structure on $V$, then the associated $\mathbb{k}[G]$-comodule structure is $\widehat{\chi}=(\mathrm{id} \otimes \pi) \chi$, where $\pi: \mathbb{k}[M] \rightarrow \mathbb{k}[G]$ is the restriction map that can be viewed as an inclusion.

Moreover, the density condition guarantees that the category $\operatorname{Rep}(M)$ of polynomial $M$-modules is a full subcategory of $\operatorname{Rep}(G)$, the category of rational $G$ modules. In particular, if $G$ is reductive, then $\operatorname{Rep}(M)$ is a semisimple category. 
The following definition is relevant to pin down the difference between representations of $G$ and of $M$.

Definition 2.4. In the situation above, a rational representation $\theta$ of $G$ is said to be polynomial (with respect to $M$ ) if there exists a polynomial representation $\chi$ of $M$ with the property that $\hat{\chi}=\theta$. Equivalently, a rational representation of $G$ is polynomial if the action of $G$ can be extended to an action of $M$; this extension is clearly unique.

In the case that $G$ is an affine algebraic group and $M$ is a monoid equipped with a polynomial character $\rho: M \rightarrow \mathbb{k}$ with the property that $G=G(M)=$ $M_{\rho}=\{m \in M: \rho(m) \neq 0\}$ (see Observation 2.2), more can be said concerning the relationship between the rational and polynomial representations of $G$, as the following easy lemma shows.

Lemma 2.5. In the situation above, if $\chi: V \rightarrow V \otimes \mathbb{k}[G]$ is a rational finitedimensional representation of $G$, there is an exponent $n \geq 0$ with the property that the rational representation of $G,\left.\rho^{n}\right|_{G} \chi: V \rightarrow V \otimes \mathbb{k}[G]$ is polynomial.

\section{Generalized Cayley's $\Omega$-Processes}

Let $M$ be an affine algebraic monoid and assume that $\lambda$ is a nontrivial character of $M$. In this section we will not need to use the density condition.

Definition 3.1. An $\Omega$-process (associated to $\lambda$ ) is a nonzero linear operator $\Omega$ : $\mathbb{k}[M] \rightarrow \mathbb{k}[M]$ such that for all $f \in \mathbb{k}[M]$ and $m \in M$ :

$$
\Omega(f \cdot m)=\lambda(m) \Omega(f) \cdot m ; \Omega(m \cdot f)=\lambda(m) m \cdot \Omega(f) .
$$

We will usually abbreviate and call an $\Omega$-process simply a process.

In classical nomenclature the above definition is called the "first rule of the $\Omega$-process".

Observation 3.2. Clearly, if $\Omega$ is a process associated to $\lambda$, then $\Omega^{r}$ is a process associated to $\lambda^{r}$.

Example 3.3 (The classical $\Omega$-process). Let $M=\mathrm{M}_{n}(\mathbb{k})$. Then the differential operator of order $n, \Omega: \mathbb{k}\left[x_{11}, \ldots, x_{n n}\right] \rightarrow \mathbb{k}\left[x_{11}, \ldots, x_{n n}\right]$, given by

$$
\Omega(f)=\sum_{\sigma \in \mathcal{S}_{n}} \operatorname{sg}(\sigma) \frac{\partial^{n}}{\partial x_{1 \sigma(1)} \cdots \partial x_{n \sigma(n)}}(f)
$$

is an $\Omega$-process; see [6] for a modern presentation.

Lemma 3.4. In the notation above, let $\mu \in \mathcal{X}(M)$ be a polynomial character of the monoid, and $g, h \in \mathbb{k}[M]$ a right (respectively left) $M$-semi-invariant of weight $\mu^{s}$. Then for all $m \in M, f \in \mathbb{k}[M]$ :

$$
\Omega^{r}((f \cdot m) g) \mu^{s}(m)=\Omega^{r}((f g) \cdot m)=\lambda^{r}(m) \Omega^{r}(f g) \cdot m
$$

and

$$
\Omega^{r}((m \cdot f) h) \mu^{s}(m)=\Omega^{r}(m \cdot(f h))=\lambda^{r}(m) m \cdot \Omega^{r}(f h) .
$$

In particular,

$$
\lambda^{r}(m) m \cdot \Omega^{r}\left(\mu^{s}\right)=\Omega^{r}\left(\mu^{s}\right) \mu^{s}(m)=\lambda^{r}(m) \Omega^{r}\left(\mu^{s}\right) \cdot m \quad \forall r, s \geq 1,
$$


and

$$
\Omega^{r}\left(\mu^{s}\right)(1) \mu^{s}(m)=\lambda^{r}(m) \Omega^{r}\left(\mu^{s}\right)(m) \quad \forall t, s \geq 1 .
$$

In other words, $\Omega^{r}\left(\mu^{s}\right)(1) \mu^{s}=\lambda^{r} \Omega^{r}\left(\mu^{s}\right)$.

Proof. It follows easily from the definition of a process, and the fact that $\Omega^{r}$ is a process for $\lambda^{r}$.

Lemma 3.5. In the notation above, if $M$ is irreducible we have that $\Omega\left(\lambda^{s}\right) \cdot m=$ $\Omega\left(\lambda^{s}\right) \lambda^{s-1}(m)$ and $m \cdot \Omega\left(\lambda^{s}\right)=\Omega\left(\lambda^{s}\right) \lambda^{s-1}(m)$. Moreover, $\Omega\left(\lambda^{s}\right)=\Omega\left(\lambda^{s}\right)(1) \lambda^{s-1}=$ $\alpha_{1, s} \lambda^{s-1}$ with $\alpha_{1, s}=\Omega\left(\lambda^{s}\right)(1) \in \mathbb{k}$.

Observation 3.6. In particular, if we call $\Omega^{r}\left(\lambda^{s}\right)(1)=\alpha_{r, s}$, we deduce that

$$
\Omega^{r}\left(\lambda^{s}\right) \lambda^{r}=\alpha_{r, s} \lambda^{s} .
$$

From $\Omega\left(\lambda^{s}\right)=\alpha_{1, s} \lambda^{s-1}$, we deduce that $\Omega^{2}\left(\lambda^{s}\right)=\alpha_{1, s} \alpha_{1, s-1} \lambda^{s-2}$ if $s \geq 2$, and in general if we call $c_{s}=\alpha_{1, s} \alpha_{1, s-1} \ldots \alpha_{1,1}$, then $\Omega^{s}\left(\lambda^{s}\right)=c_{s} \in \mathbb{k}$.

Definition 3.7. In the situation above we say that the $\Omega$-process is proper if the elements $c_{s} \in \mathbb{k}$ are not zero for all $s \geq 1$.

Observation 3.8. Let $\mu \in \mathcal{X}(M), g, h \in \mathbb{k}[M]$ be a right (respectively left) $M$ semi-invariant of weight $\mu^{s}$ and $f \in \mathbb{k}[M]$. If we write $f \cdot m=\sum f_{1}(m) f_{2}$, we have that

$$
\Omega^{r}((f \cdot m) g) \mu^{s}(m)=\left(\sum f_{1}(m) \Omega^{r}\left(f_{2} g\right)\right) \mu^{s}(m)=\lambda^{r}(m) \Omega^{r}(f g) \cdot m .
$$

Evaluating the above equality at 0 we deduce that

$$
\Omega^{r}(f g)(0) \lambda^{r}=\left(\sum f_{1} \Omega^{r}\left(f_{2} g\right)(0)\right) \mu^{s} .
$$

Similarly, $\Omega^{r}(f h)(0) \lambda^{r}=\left(\sum \Omega^{r}\left(f_{1} h\right)(0) f_{2}\right) \mu^{s}$.

In particular, if $g=h=\lambda=\mu$ we have that

$$
\Omega^{r}\left(f \lambda^{s}\right)(0) \lambda^{r}=\lambda^{s} \sum \Omega^{r}\left(f_{1} \lambda^{s}\right)(0) f_{2}=\lambda^{s} \sum f_{1} \Omega^{r}\left(f_{2} \lambda^{s}\right)(0) .
$$

Moreover, if $r=s=1$, then, if $M$ is irreducible, $\Omega(f \lambda)(0)=\sum f_{1} \Omega\left(f_{2} \lambda\right)(0)=$ $\sum \Omega\left(f_{1} \lambda\right)(0) f_{2}$.

\section{Existence of CAyley's $\Omega$-PRocesses}

In this section we describe, in the case of an irreducible reductive monoid $M$, all the $\Omega$-processes associated to a polynomial character $\lambda \in \mathcal{X}(M)$.

Observation 4.1. The action of $G \times G$ on $M,(a, b) \cdot m=a m b^{-1}$ induces a structure of a rational $(G \times G)$-module on $\mathbb{k}[M]$. It follows from the Peter-Weyl theorem that there is a submonoid $C_{M} \subset \mathcal{X}_{+}(T)$ such that

$$
\mathbb{k}[M]=\sum_{\mu \in C_{M}} V_{\mu} \otimes V_{\mu}^{*} \subset \sum_{\mu \in \mathcal{X}_{+}(T)} V_{\mu} \otimes V_{\mu}^{*}=\mathbb{k}[G] .
$$

Recall that $V_{\mu} \otimes V_{\mu}^{*}$ is a simple $(G \times G)$-module of weight $(\mu,-\mu)$.

Theorem 4.2. Let $\mu \in C_{M}$ and $f \in \mathbb{k}[M]$ be $a\left(B \times B^{-}\right)$-semi-invariant of weight $(\mu,-\mu)$. Then $\Omega(f)$ is a $\left(B \times B^{-}\right)$-semi-invariant of weight $(\mu-\lambda, \lambda-\mu)$, where $\Omega$ is a process associated to $\lambda$. 
Proof. In the same manner as before, from the definition of a process we deduce that for all $b_{1} \in B$ and $b_{2} \in B^{-}$,

$$
\begin{aligned}
\left(b_{1}, b_{2}\right) \cdot \Omega(f) \lambda\left(b_{1}\right) \lambda\left(b_{2}^{-1}\right) & =\left(b_{1} \cdot \Omega(f) \cdot b_{2}^{-1}\right) \lambda\left(b_{1}\right) \lambda\left(b_{2}^{-1}\right) \\
& =\Omega\left(\left(b_{1}, b_{2}\right) \cdot f\right)=\mu\left(b_{1}\right) \mu\left(b_{2}^{-1}\right) \Omega(f) .
\end{aligned}
$$

Corollary 4.3. Let $\Omega$ be a process for $M$ associated to a polynomial character $\lambda$, and let $f \in \mathbb{k}[M]$ be a semi-invariant of weight $(\mu,-\mu)$. If $\mu-\lambda \notin C_{M}$, then $\Omega(f)=0$.

Theorem 4.4. Let $M$ be an irreducible reductive monoid, $\lambda \in \mathcal{X}(M)$ and $\Omega$ a process associated to $\lambda$. Then $\Omega=\bigoplus_{\mu \in C_{M}} \frac{a_{\mu}}{\lambda} \operatorname{Id}_{\mu}$, where $\operatorname{Id}_{\mu}$ is the identity map of $V_{\mu} \otimes V_{\mu}^{*}$ and $a_{\mu} \in \mathbb{k}$. Moreover, if $\mu-\lambda \notin C_{M}$, then $a_{\mu}=0$. In particular, $\Omega$ is proper if and only if $a_{\lambda^{s}} \neq 0$ for all $s \geq 0$. Conversely, given $\lambda \in \mathcal{X}(M)$, if we define $\Omega$ as above with $a_{\mu}=0$ for $\mu-\lambda \notin C_{M}$, then $\Omega$ is a process associated to $\lambda$.

Proof. From the definition of a process, it is clear that $\Omega$ stabilizes the $(G \times G)$ submodules of $M$, and hence $\Omega$ is characterized by its restrictions to $V_{\mu} \otimes V_{\mu}^{*}$, $\mu \in C_{M}$.

If $f_{\mu} \in V_{\mu} \otimes V_{\mu}^{*}$ is a $\left(B \times B^{-}\right)$-semi-invariant, then it follows from Theorem 4.2 that $\Omega\left(f_{\mu}\right)$ is also a semi-invariant, of weight $(\mu-\lambda, \lambda-\mu)$. Hence, $\Omega\left(f_{\mu}\right)=a_{\mu} \frac{f_{\mu}}{\lambda}$, where $a_{\mu} \in \mathbb{k}$ is necessarily zero if $\mu-\lambda \notin C_{M}$.

Again from the definition of a process, it follows that if $s, t \in G$, then

$$
\begin{aligned}
\Omega\left((s, t) \cdot f_{\mu}\right) & =\Omega\left(s \cdot f_{\mu} \cdot t^{-1}\right)=\lambda\left(t^{-1}\right) \lambda(s)\left(s \cdot \Omega\left(f_{\mu}\right) \cdot t^{-1}\right) \\
& =a_{\mu} \lambda\left(t^{-1}\right) \lambda(s) \frac{s \cdot f_{\mu} \cdot t^{-1}}{s \cdot \lambda \cdot t^{-1}}=a_{\mu} \frac{(s, t) \cdot f_{\mu}}{\lambda} .
\end{aligned}
$$

It follows from Corollary 4.3 that if $\mu-\lambda \notin C_{M}$, then $a_{\mu}=0$.

The assertion about the properness of the $\Omega$-process follows directly from the above definition.

It is easy to show the converse, i.e., any election of $a_{\mu} \in \mathbb{k}$, with $\mu-\lambda \in C_{M}$, is adequate in order to obtain an $\Omega$-process.

\section{Finite generation of invariants}

In this section we show that the existence of a proper process guarantees the finite generation of the rings of invariants corresponding to linear actions.

Let $M$ be a linear algebraic monoid with zero and dense unit group $G$. Assume that $\Omega$ is a proper process with associated character $\lambda$.

If $V$ is a polynomial $M$-module, and $r, s$ are positive integers, we define the map $I_{r, s}: V \rightarrow V$ as the composition:

$$
I_{r, s}: V \stackrel{\chi}{\longrightarrow} V \otimes \mathbb{k}[M] \stackrel{\text { id } \otimes \lambda^{s}}{\longrightarrow} V \otimes \mathbb{k}[M] \stackrel{\text { id } \otimes \Omega^{r}}{\longrightarrow} V \otimes \mathbb{k}[M] \stackrel{\text { id } \otimes \varepsilon_{0}}{\longrightarrow} V \otimes \mathbb{k} \cong V
$$

where $\varepsilon_{0}: \mathbb{k}[M] \rightarrow \mathbb{k}$ is the evaluation at $0 \in M$. In terms of Sweedler's notation, $I_{r, s}(v)=\sum v_{0} \Omega^{r}\left(\lambda^{s} v_{1}\right)(0)$, where $\chi(v)=\sum v_{0} \otimes v_{1} \in V \otimes \mathbb{k}[M]$.

The results of the theorem that follows were known in the classical literature as the "second rule of the $\Omega$-process". 
Theorem 5.1. In the situation above, let $V$ be a polynomial $G$-module with structure $\theta: V \rightarrow V \otimes \mathbb{k}[G]$ and consider the associated $M$-module structure $\chi: V \rightarrow$ $V \otimes \mathbb{k}[M]$ such that $\theta=(\mathrm{id} \otimes \pi) \chi$. Then $c_{r}^{-1} I_{r, s}: V \rightarrow V$ is an $M$-equivariant projection onto the space of the $G$-semi-invariants of weight $\lambda^{r-s}$, where $I_{r, s}$ is as above and $c_{r}=\Omega^{r}\left(\lambda^{r}\right) \in \mathbb{k}$.

Proof. First we prove that the map $I_{r, s}$ is an $M$-morphism, i.e., that the diagram

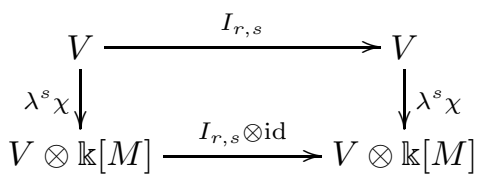

is commutative.

$$
\begin{aligned}
& \text { Indeed, } \begin{aligned}
\lambda^{s} \chi\left(I_{r, s}(v)\right) & =\lambda^{s} \sum v_{0} \otimes v_{1} \Omega^{r}\left(\lambda^{s} v_{2}\right)(0)=\sum v_{0} \Omega^{r}\left(\lambda^{s} v_{1}\right)(0) \otimes \lambda^{r} . \text { Also, } \\
\left(I_{r, s} \otimes \mathrm{id}\right)\left(\lambda^{s} \chi(v)\right) & =\sum I_{r, s}\left(v_{0}\right) \otimes \lambda^{s} v_{1}=\sum v_{0} \otimes \lambda^{s} \Omega^{r}\left(\lambda^{s} v_{1}\right)(0) v_{2} \\
& =\sum v_{0} \otimes \Omega^{r}\left(\lambda^{s} v_{1}\right)(0) \lambda^{r}=\sum v_{0} \Omega^{r}\left(\lambda^{s} v_{1}\right)(0) \otimes \lambda^{r}
\end{aligned}
\end{aligned}
$$

Applying id $\otimes \pi$ to the equality $\lambda^{s} \chi\left(I_{r, s}(v)\right)=\lambda^{r}\left(I_{r, s}(v) \otimes 1\right)$ we obtain that $\left.\lambda^{s}\right|_{G} \theta\left(I_{r, s}(v)\right)=\left.I_{r, s}(v) \otimes \lambda^{r}\right|_{G}$, and hence we conclude that $I_{r, s}$ is a $G$-semi-invariant of weight $\lambda^{r-s}$.

Moreover, if $v \in V$ is $G$-semi-invariant of weight $\lambda^{r-s}$, then $\theta(v)=\left.v \otimes \lambda^{r-s}\right|_{G}$. Hence, $\sum v_{0} \otimes \lambda^{s} v_{1}=\lambda^{s} \chi(v)=v \otimes \lambda^{r}$ and applying id $\otimes \Omega^{r}$ we deduce that $I_{r, s}(v)=$ $c_{r} v$.

The proof of the theorem that follows is closely related to the standard methods introduced in [2]; see also 6] for a modern presentation in the case of $\mathrm{GL}_{n}$.

Theorem 5.2. Let $M$ be a linear algebraic monoid with 0 and assume that for some polynomial character $\lambda: M \rightarrow \mathbb{k}, G=G(M)=M_{\lambda}$. Assume moreover that $M$ admits a proper $\Omega$-process associated to $\lambda$. If $V$ is a finite-dimensional rational $G$-module, then the ring of invariants $\mathrm{S}(V)^{G}$ is finitely generated.

Proof. Let $I=\left\langle\mathrm{S}_{+}(V)^{G}\right\rangle \subset \mathrm{S}(V)$ be the ideal of $\mathrm{S}(V)$ generated by the homogeneous invariants of positive degree. Let $\left\{\xi_{1}, \ldots, \xi_{t}\right\} \subset \mathrm{S}_{+}(V)^{G}$ be a finite number of homogeneous ideal generators of $I$, and call $d_{i}$ the degrees of the $\xi_{i}$ for $i=1, \ldots, t$. Evidently $\mathbb{k}\left[\xi_{1}, \ldots, \xi_{t}\right] \subset \mathrm{S}(V)^{G}$; we will prove by induction on $d>0$ that $\mathrm{S}_{d}(V)^{G} \subset \mathbb{k}\left[\xi_{1}, \ldots, \xi_{t}\right]$.

Assume that for all $e<d, \mathrm{~S}_{e}(V)^{G} \subset \mathbb{k}\left[\xi_{1}, \ldots, \xi_{t}\right]$ and take $\xi \in \mathrm{S}_{d}(V)^{G} \subset I$. Then, we can find homogeneous elements $f_{i} \in \mathrm{S}_{e_{i}}(V)$ such that $\xi=\sum f_{i} \xi_{i}$, with $d=e_{i}+d_{i}$, and $e_{i}<d$ for $i=1, \ldots, t$.

Call $\theta: V \rightarrow V \otimes \mathbb{k}[G]$ the comodule structure on $V$. As we already observed, there exist $n \geq 0$ and $\chi: V \rightarrow V \otimes \mathbb{k}[M]$, a polynomial $\mathbb{k}[M]$-comodule structure on $V$, such that $\lambda^{n} \theta=($ id $\otimes \pi) \chi: V \rightarrow V \otimes \mathbb{k}[G]$. If we call $\theta_{r}: \mathrm{S}_{r}(V) \rightarrow \mathrm{S}_{r}(V) \otimes \mathbb{k}[G]$ the comodule structure induced by $\theta$ on $\mathrm{S}_{r}(V)$, then $\lambda^{n r} \theta_{r}$ is polynomial. 
Applying $\theta_{d}$ to the equality $\xi=\sum f_{i} \xi_{i}$ we obtain: $\xi \otimes 1=\sum_{i=1}^{t} f_{i 0} \xi_{i} \otimes f_{i 1}$ and $\xi \otimes \lambda^{n d}=\sum_{i=1}^{t} f_{i 0} \xi_{i} \otimes \lambda^{n d_{i}} \lambda^{n e_{i}} f_{i 1}$.

Call $e_{i}^{\prime}$ the minimal exponent such that the coaction $\lambda^{n e_{i}^{\prime}} \theta_{e_{i}}: \mathrm{S}_{e_{i}}(V) \rightarrow \mathrm{S}_{e_{i}}(V) \otimes$ $\mathbb{k}[G]$ is polynomial and write $\lambda^{n e_{i}^{\prime}} \theta_{e_{i}}\left(f_{i}\right)=\sum f_{i 0} \otimes g_{i 1}$ with $f_{i 0} \in \mathrm{S}_{e_{i}}(V)$ and $g_{i 1} \in \mathbb{k}[M]$. Then $e_{i}^{\prime} \leq e_{i}$ and we have that $\xi \otimes \lambda^{n d}=\sum_{i=1}^{t} f_{i 0} \xi_{i} \otimes \lambda^{n d_{i}^{\prime}} g_{i 1}$, with $d_{i}^{\prime} \geq d_{i}$, where $d_{i}^{\prime}=d-e_{i}^{\prime}$.

Applying $\Omega^{n d}$, then evaluating at zero and recalling that $\Omega^{n d}\left(\lambda^{n d}\right)=c_{n d} \in \mathbb{k}^{*}$, we obtain

$$
c_{n d} \xi=\sum_{i=1}^{t} f_{i 0} \Omega^{n d}\left(\lambda^{n d_{i}^{\prime}} g_{i 1}\right)(0) \xi_{i}=\sum_{i=1}^{t} I_{n d, n d_{i}^{\prime}}\left(f_{i}\right) \xi_{i},
$$

where $I_{n d, n d_{i}^{\prime}}$ is as in Theorem 5.1 where it is proved that it is a semi-invariant of weight $\lambda^{n e_{i}^{\prime}}$.

As all $\xi, \xi_{i}, i=1, \ldots, t$ are in fact invariants, we conclude that $I_{n d, n d_{i}^{\prime}}\left(f_{i}\right) \in$ $\mathrm{S}_{e_{i}}^{G}(V) \subset \mathbb{k}\left[\xi_{1}, \ldots, \xi_{t}\right]$. Then as $c_{n d} \neq 0$, we have that $\xi \in \mathbb{k}\left[\xi_{1}, \ldots, \xi_{t}\right]$ and the proof is finished.

We finish by presenting a proof of the classical result on the finite generation of the ring of linear invariants of an arbitrary reductive group, that uses the above theorem as well as the construction of proper processes for reductive monoids.

Theorem 5.3. Let $G$ be a reductive group and $V$ a finite-dimensional rational $G$-module. Then $\mathrm{S}(V)^{G}$ is a finitely generated $\mathbb{k}$-algebra.

Proof. It is only necessary to deal with the case where $G$ is connected. Indeed, $\mathrm{S}(V)^{G}=\left(\mathrm{S}(V)^{G_{1}}\right)^{G / G_{1}}$, where $G_{1}$ is the connected component of the identity.

If $G$ is connected, let $W$ be a faithful finite-dimensional representation of $G$ containing the trivial representation. Then $G \subset \mathrm{GL}(W)$ and $\mathbb{k}^{*} \operatorname{Id} \cap G=\{\operatorname{Id}\}$. Hence $G^{\prime}=\left(\mathbb{k}^{*} \mathrm{Id}\right) G \cong \mathbb{k}^{*} \times G$ is a connected reductive group and $M=\overline{G^{\prime}} \subset$ $\operatorname{End}(W)$ is an irreducible reductive monoid with zero whose unit group is $G^{\prime}$ (see [5, Prop. 1]).

If we extend the action of $G$ on $V$ to an action of $G^{\prime}$ by $(z, g) \cdot v=g \cdot v$, then using Observation 2.2, Theorem 4.4, and Theorem 5.2 we conclude our result.

\section{ACKNOWLEDGEMENTS}

The authors thank the referee for her/his suggestions concerning generalizations as well as simplifications of some of the proofs and results.

\section{REFERENCES}

[1] W. Ferrer Santos and A. Rittatore, Actions and Invariants of Algebraic Groups. Series: Pure and Applied Mathematics, 268, Dekker-CRC Press, Florida, (2005). MR2138858 (2006c:14067)

[2] D. Hilbert, Über die Theorie der algebraischen Formen. Matematische Annalen, 36 (1890) pages 473-534. MR1510634

[3] M. S. Putcha, Linear Algebraic Monoids, London Math. Soc. Lect. Notes 133, Cambridge Univ. Press, 1988. MR0964690 (90a:20003)

[4] L. Renner, Linear Algebraic Monoids. Encyclopaedia of Math. Sciences 134, Invariant Theory V., Springer Verlag, 2005. MR.2134980 (2006a:20002) 
[5] A. Rittatore, Algebraic Monoids and group embeddings. Trans. Groups Vol 3, No 4 (1998), pages 375-396. MR1657536 (2000a:14056)

[6] B. Sturmfels, Algorithms in invariant theory. Texts and Monographs in Symbolic Computation. Springer-Verlag, 1993. MR1255980 (94m:13004)

Facultad de Ciencias, Universidad de la República, Iguá 4225, 11400 Montevideo, URUGUAY

E-mail address: wrferrer@cmat.edu.uy

Facultad de Ciencias, Universidad de la República, Iguá 4225, 11400 Montevideo, URUGUAY

E-mail address: alvaro@cmat.edu.uy 